\title{
Personal genome testing in medical education: student experiences with genotyping in the classroom
}

\author{
Simone Lucia Vernez ${ }^{1}$, Keyan Salari ${ }^{2}$, Kelly E Ormond ${ }^{1,2}$ and Sandra Soo-Jin Lee L,3* $^{1,}$
}

\begin{abstract}
Background: Direct-to-consumer (DTC) personal genotyping services are beginning to be adopted by educational institutions as pedagogical tools for learning about human genetics. However, there is little known about student reactions to such testing. This study investigated student experiences and attitudes towards DTC personal genome testing.

Methods: Individual interviews were conducted with students who chose to undergo personal genotyping in the context of an elective genetics course. Ten medical and graduate students were interviewed before genotyping occurred, and at 2 weeks and 6 months after receiving their genotype results. Qualitative analysis of interview transcripts assessed the expectations and experiences of students who underwent personal genotyping, how they interpreted and applied their results; how the testing affected the quality of their learning during the course, and what were their perceived needs for support.
\end{abstract}

Results: Students stated that personal genotyping enhanced their engagement with the course content. Although students expressed skepticism over the clinical utility of some test results, they expressed significant enthusiasm immediately after receiving their personal genetic analysis, and were particularly interested in results such as drug response and carrier testing. However, few reported making behavioral changes or following up on specific results through a healthcare provider. Students did not report utilizing genetic counseling, despite feeling strongly that the 'general public' would need these services. In follow-up interviews, students exhibited poor recall on details of the consent and biobanking agreements, but expressed little regret over their decision to undergo genotyping. Students reported mining their raw genetic data, and conveyed a need for further consultation support in their exploration of genetic variants.

Conclusions: Personal genotyping may improve students' self-reported motivation and engagement with course material. However, consultative support that is different from traditional genetic counseling will be necessary to support students. Before incorporating personal genotyping into coursework, institutions should lead multi-disciplinary discussion to anticipate issues and incorporate teaching mechanisms that engage the ethical, legal, and social implications of personal genotyping, including addressing those found in this study, to go beyond what is offered by commercial providers.

\section{Background}

Direct-to-consumer (DTC) personal genomics companies provide public access to individualized genetic testing outside the context of traditional healthcare systems. For a fee, consumers may submit a saliva specimen for analysis to obtain genetic risk information on a broad

\footnotetext{
* Correspondence: sandra.lee@stanford.edu

'Stanford Center for Biomedical Ethics, 1215 Welch Road, Mod A, Stanford,

CA 94305 USA

Full list of author information is available at the end of the article
}

spectrum of health conditions and behavioral traits. Such services have prompted concerns that consumers will approach their physicians for assistance with interpretation and/or incorporation of individual results into their healthcare management $[1,2]$. Furthermore, owing to the declining costs of personal genomic testing, some hospitals in the USA and other countries have begun to introduce genomic analysis to make pharmacogenetic testing available to patients [3-5]. However, physicians are often ill equipped to interpret or utilize these genetic

\section{Biomed Central}


profiles, prompting calls to educate healthcare providers on genome-wide association studies and on medical genetics more generally [6-10]. Consequently, several institutions have begun to consider, and in some cases offer, courses that incorporate genomic testing of students as an innovative pedagogical approach to teaching human genetics [11-14].

Although there is widespread agreement about the need for improved medical education in genomic medicine, there are few models for creating effective mechanisms and content within existing curricula to achieve these goals [15-18]. Recently, personal genotyping has gained attention as a way to increase medical professionals' knowledge of emerging genetic technologies and scientific discovery, and to engender excitement and motivation among students as a way of encouraging them to learn and engage with genetics [7-11,19,20].

In 2009, a medical school elective course was proposed at Stanford University to expose physician-scientist trainees to the design, use, and interpretation of genetic studies of human populations and diseases [14]. The course, GENE 210 'Genomics and Personalized Medicine' aimed to educate students on the analysis and interpretation of individual genome data as it relates to disease risk, pharmacogenomics, and human ancestry [21]. In an effort to augment the learning environment, course instructors proposed that students should have the option to use their own genotype data in classroom exercises. To review this new approach, a multi-disciplinary task force consisting of faculty members in genetics, genetic counseling, law, ethics, education, and clinical departments, convened to discuss the proposal and to explore the risks of incorporating DTC personal genetic testing into the elective medical school course $[14,19]$. Through this process, the university task force identified 8 potential ethical challenges and their possible solutions. As described in Figure 1, these issues were confidentiality, conflict of interest, risk of coercion, informational risks, informed decision-making, financial accessibility, genetic counseling, and unknown issues due to the experimental nature of the new course [19]. The task force made several recommendations to address these concerns, such as offering the option for carefully subsidized testing through two different companies (23andMe, Inc. or Navigenics), establishing a strict policy of anonymity, offering free genetic counseling, and obtaining approval for use of human subjects for the empirical study of the course method [19]. These recommendations were implemented when the course was first offered during the summer quarter of 2010.

The course at Stanford is now one of several across the USA that has incorporated personal genotyping as a pedagogical tool in teaching human genetics, reflecting growing interest in institutions for such approaches $[7-11,19,20]$. However, as yet, there have been no empirical studies of student experience with this method. In this study, we explored the perspectives of students who had undergone personal genotyping in a course context, by conducting a series of individual interviews with students enrolled in GENE 210 before and after genotyping. Our specific research questions were as follows. 1) What are the expectations of students choosing to undergo personal genotyping? 2) How do students interpret and apply their genetic results? 3 ) What do students understand to be their relationship with DTC genetic testing commercial providers? 4) What type of support, if any, do students need?

\section{Methods}

\section{Ethics approval}

The research conformed to the Declaration of Helsinki's Ethical Principles for Medical Research Involving Human Subjects, and was approved by the Stanford Institutional Review Board. Students gave written and oral informed consent to participate in the study.

\section{Participants}

We recruited study participants from the 46 students enrolled in GENE 210: 'Genomics and Personalized Medicine' at Stanford University School of Medicine offered in the summer of $2010 \mathrm{http} / / /$ gene210.stanford. edu/. During the 8-week course, students analyzed whole-genome single nucleotide polymorphism (SNP) data, and were given a choice of using their own data or publicly available genotype data from 12 HapMap participants.

To recruit participants, we distributed an email announcement that included details of the study to registered enrollees at the beginning of the course, and instructors announced information about the study in class. Enrollment in the study was limited to students who elected to use the personal genotyping offered through the course, who were over the age of 18 years old, and who agreed to participate in open ended, indepth individual interviews. The identities of student participants were kept confidential. No instructors were involved in the enrollment of study participants, and the decision to participate was strictly voluntary and not linked in any way to student evaluations or grades in the course.

\section{Interviews}

Students recruited for this study participated in three indepth individual interviews (with author SL), each lasting approximately 90 to 120 minutes. The first interview occurred before students received their genotyping results, followed by a second interview 2 weeks after receiving their results, and a final interview approximately 6 months later. 
1. Confidentiality of the personal genomic and health information of the students.

2. Conflicts of Interest for faculty members associated with the proposed course and who also sat on the boards of testing companies.

3. Risk for Coercion of students electing to undergo testing for fear of disappointing their instructors or due to peer pressure.

4. Informational Risks to family members of students from personal genomic information.

5. Informed Decision-making that ensured the autonomy of students and full comprehension of the risks and benefits.

6. Financial Accessibility in determining the subsidized cost of the genotyping ensuring thoughtful decision-making and affordability for students.

7. Genetic Counseling Needs of students to interpret test results.

8. Experimental Nature of the Course Offering where students were participants in the assessment of genotyping as an effective educational tool.

Figure 1 Issues addressed by the university task force review.

Interviews included questions probing student perspectives on course expectations, understanding of informed consent, confidentiality of participant identity and genotype data, interpretation and application of test results, experiences sharing genotype data, and attitudes towards the pedagogical value of personal genotyping.

\section{Data analysis}

Analysis of interview data was based on the principles of grounded theory, which offers systematic procedures that move from description to analysis, and enables theories to be developed inductively. Analysis of interview data began with open coding, using the qualitative software program MAXQDA (VERBI GmBH, Berlin, Germany). This inductive method examines phenomena within the interview text, and categorizes statements through codes that are developed within a larger framework. In addition, selective coding of the data, focusing on categories and including behavioral changes, informed consent, and personal utility, was analyzed for themes across the three sets of interviews. Intra-coder and inter-coder reliability was maximized in several ways. Differences were reconciled to establish an initial coding scheme. Periodic joint coding of data ensured continued inter-coder reliability. Approximately 10\% of the data were cross-coded (by author SL) and re-coded (author SV) to ensure intra-coder consistency over time. Any coding differences encountered were discussed and reconciled through consultation with the research team.

\section{Results}

Participant characteristics are described in Table 1; 50\% were female and self-identified as Caucasian. Because GENE 210 was a graduate-level elective course, all participants were advanced students, with three participants enrolled in clinical programs (medical school or clinical fellowship) and seven enrolled in biological sciences programs (graduate school or post-doctoral fellowship). All participants chose the personal genotyping offered through 23andMe, Inc. Significant changes in attitudes and reported reactions to genotyping experiences

\section{Table 1 Participant characteristics}

\begin{tabular}{lc}
\hline Characteristics & No. (\%) \\
\hline Female gender & $5(50.0)$ \\
Program & \\
$\quad$ Medical (MD, clinical resident/Fellow) & $4(40.0)$ \\
$\quad$ Graduate (PhD, post-doctoral Fellow) & $6(60.0)$ \\
Caucasian & $5(50.0)$ \\
\hline
\end{tabular}


between the initial interviews and those conducted 6 months later were minimal. The findings reported here reflect the major themes that emerged from the three sets of interviews.

\section{Student perspectives on the pedagogical value of genotyping}

Students overwhelmingly felt that using their own genotype data in the context of the course was personally motivating in learning the course material. This sentiment was expressed throughout the three sets of interviews. One graduate student reflected on his experience by emphasizing the value of testing as a pedagogical tool.

'I think people will be more motivated to do a lot of exercises in class with their own data and therefore, once we got our own data, a lot of people were excited about it and were happy to use their own data for the other exercises. And I even went back and redid some of the old stuff, based on my data. So I think there was definitely a benefit... I don't think there's anything specific that would make the education less valuable by using the dummy datasets they provided. I think there's a more personal edge to it when you're using your own data, so there's more motivation to learn about it.'

Moreover, students training in medicine felt that their testing experience provided them with valuable insight into what patients who elect to undergo personal genetic testing might experience when they see their test results. One medical student reflected this by stating,

'I think that getting personally genotyped is really important because it is easy to tell anyone, 'oh, yeah, you should get genotyped!' But if you haven't gone through it - the personal feelings, emotions, and thought process behind it - I feel like it's hard to tell someone else to do it. It's hard as a medical doctor. How can you recommend that to your patients if you haven't actually thought about it, because it's such an emotional, personal thing. So I think it's really important to take this course.'

Students emphasized that personal genotyping promoted their engagement with the course material. Students described the benefit of reflecting on the social, ethical, and policy implications of genetic testing presented in lectures and class discussions during the course. Participants reported thinking more deeply about the risks and benefits related to testing as a result of their experience in the class. For example, issues of privacy and anonymity of genotype data were discussed by several students.

'We discussed this in class - that this is a privacy issue and that nothing is more unique than your genotype. It's your fingerprint. There are people out there right now who are saying that they can figure out where people are from, within a hundred kilometers, based on their genotype, based on just 550,000 SNPs. And I think that's concerning about genotyping data.'

They also described having a greater understanding of the social issues related to testing patients than the general population, articulating the potential value of personal experience with genotyping in bridging the gap between healthcare providers and patients.

\section{Student attitudes towards clinical utility and application of genotyping results}

Despite the strong views of participants that self-testing promoted enhanced learning of course material, most students expressed skepticism over the clinical utility of their results related to risk prediction for complex diseases (for example, heart disease, cancer, diabetes) and were dismissive of results related to behavioral traits, such as cognitive ability and athleticism. Conversely, participants did state that genetic results related to drug response and to carrier traits offered more value. For example, every participant identified drug-response information, such as sensitivity to warfarin, as potentially useful, and noted the practical personal utility of carrier testing. During her interview 2 weeks after receiving her test results, one graduate student described the potential actionability of learning she is a carrier for a monogenic disease:

'Knowing that I'm a carrier for this is something... it makes things much easier to deal with, because you can actually do something. It's real easy to get tested for it, and you can actually take corrective steps if you want to. You can get your partner tested, you can do IVF if you're really worried about it...'

When asked if the participant would ask her partner to be tested for the condition for which she was found to be a carrier, she answered that her partner was planning to be tested. When asked about the partner's decision in the follow-up interview 6 months later, the participant said that her partner had not yet been tested, explaining,

'He's probably going to get tested for it. I am not sure when. It seemed like a big deal for us when I 
first got my results but, honestly, we haven't thought much about it lately. I guess you could say it fell down on the list of things to do.'

Carrier-testing results were received with great interest, more so than other test results returned to participants, and in several cases, participants reported that the test results triggered conversations between participants and their partners about the meaning of results for reproductive decisions. However, in the 6-month follow-up interviews, there was little indication that carrier or pharmacogenetic testing prompted specific actions for participants. Given that students were not taking the relevant drugs or making imminent family-planning decisions at the time of the study, it is unclear whether the 6-month time frame of the study was sufficient to determine whether testing information would eventually prompt behavioral changes or health management decisions.

Students did report intentions to make modest behavioral changes as a result of risk predictions of complex diseases and conditions. For example, 2 weeks after receiving their results, students reported plans to improve their diet, exercise more regularly, and wear sunglasses when outside. In one case, a student said she intended to consume dairy products more conscientiously in light of test results that indicated a high likelihood of lactose intolerance, in order to stave off developing the condition. However, with the exception of this last example, students did not report making significant progress on these intentions in interviews 6 months after receiving their genotype results.

\section{Student perspectives on consultative support}

Although all students were given the option of consulting independent genetic-counseling services before and after testing at no additional charge, utilization of these services was very low. Participants felt that they were well equipped to interpret their test results on their own; consequently, only one student participant expressed any interest in this offering. By contrast, participants on the whole felt strongly that genetic-counseling services would be important for the general public. One participant explained,

'Because I am a student in biosciences, I feel like I can interpret the data myself, and so in that sense I would personally resent having to go through a doctor to get my genome information. But, maybe I'm being a little paternalistic in that I think for the general public, they should go through their doctors to get information like this.'

Although only one participant had specific training in clinical genetics, none of the participants felt that genetic-counseling services should be required of students enrolled in the course.

Most of the study participants reported that they had used or planned to use the bioinformatics tools presented in the course to browse their genome for results not returned by their DTC personal genomics provider. Most commonly, students had used their raw data to assess their relative risk for conditions known in their family history, but all participants also mentioned mining their data for a broad range of reported variants, using outside sources, such as SNPedia http://www. snpedia.com/index.php/SNPedia. For example, although at the time, 23andMe Inc. did not offer analysis of the apolipoprotein E (APOE) locus, which is associated with the onset of Alzheimer's disease, nearly all of the participants had already searched for or planned to check their likely APOE status using their raw data.

Several participants said that they needed more individualized help analyzing their raw dataset when mining it for additional information. Their questions focused on technical questions about the mining process itself, and only secondarily about the clinical utility of a particular finding. One participant suggested that the ethical guidelines established to ensure confidentiality in the course impeded the students' ability to get the help they needed. He explained,

'I think there were issues with the course that could be improved. For example, one of the issues was we weren't supposed to tell any instructors that we got genotyped. So, this actually was, in some ways, really hobbling, because if you had a specific question about how to analyze data, because of a certain population structure you were observing, but you couldn't... So, because of the way the class was structured to provide complete anonymity, it basically meant that the class was not a resource for your personal genomics information.'

Several students who were eager to apply the bioinformatics tools they had learned in the course on their own data felt stymied by the inability to discuss their personal results with instructors who might help them with their analyses.

\section{Student experiences of informed consent}

When participants were asked about the details of the consent form provided online by the genetic testing services, none of the participants could accurately recall the details of the agreement. Nor could any of the participants fully describe the conditions of biobanking, an option offered by 23 andMe, Inc. to store genetic samples with the company for further study, including company-sponsored research. All but two participants 
elected for their samples to be biobanked. However, none of these individuals could accurately describe how long samples would be stored; what, if any, personal information would be stored with samples; and whether they had the option of withdrawing their samples once enrolled. One participant answered,

'... you know, I didn't read all the legal stuff about it... I guess it was... the general understanding was that they... they use it, you know, they... Honestly, I'm not sure... my name isn't necessarily tied to the studies that they'll use. I'm sure they have some information I'm associated with, but... um, and that it would be used for their private research of some kind... I don't know what that all involves, I guess (chuckles)'

Most students admitted to not reading all the legal terms and conditions regarding the 23andMe Inc. biobanking program, and were unsure of how their personal information or genetic data might be used by the company for research purposes. Although participants could not describe the content of the consent and biobanking agreements well, none of the participants expressed concern over their lack of recall. All participants stated that, given the opportunity, they would have chosen self-testing as part of the course again, expressing few regrets over their decisions.

\section{Discussion}

The advancing pace of genetic discovery has created a widening gap in medical education. To promote genetic literacy among students, several institutions are incorporating personal genotyping in the classroom $[7-11,19]$. We report here one of the first studies of student experiences with genotyping used as a pedagogical tool to teach human genetics. This study was limited to the perspectives of those students who elected to use genotyping and who had advanced training in medicine and various scientific fields (although little, if any, training in clinical genetics). Results from the pre-testing and posttesting interviews with study participants suggest that students perceived positive benefits from using their own genetic data. Participants described using their own genetic data in the classroom as personally motivating, and felt it provided insight into the patient experience. Participants valued the faculty-led discussion of the ethical and legal issues related to personal genetic testing, and stated that these allowed them to consider dimensions to their decision that they had not identified prior to the course. Our findings also indicate that the careful consideration by the university task force of how the course should be executed resulted in measures being put in place that mitigated potential problems. For example, participants specifically cited that setting the price of the testing at $\$ 99$ forced participants to carefully consider their decision in ways that a completely free test might not.

The study results also indicate a need for new models of consultative support for students who undergo selftesting. Our study findings indicate an underutilization of genetic-counseling services. Although few studies have examined consumer decisions to seek genetic counseling, this result is consistent with a survey based study conducted by Kaufman et al., [22] in which a mere $1 \%$ of participants sought out genetic counseling when interpreting their results [23]. Our study result may be explained in part by the biased perception of the students undergoing personal genotyping that, unlike the general population, they possessed sufficient knowledge to interpret their results. However, more research is needed to determine whether such sentiments are evident among other DTC personal genomics clientele.

Consistent with the few empirical studies that have examined the behavioral responses of consumers of DTC genetic testing services, our study results provide little evidence to suggest that the mere provision of genetic information alone results in widespread changes in health behaviors [2,23]. Although students did report their intention to make modest behavioral changes as a result of risk predictions of complex diseases and conditions shortly after receiving their results, none had made any clinical changes or pursued follow-up care with healthcare providers 6 months later.

The finding that many of our participants placed a high value on carrier-testing information and their relevance for reproductive decision-making suggests the need to attend to the specific concerns of student populations when genotyping is offered in coursework. Although our participants took little, if any, action based on their carrier-testing results, the age range of the study participants coincided with the age at which many students may be concerned with reproductive decisions but may not be compelled to act on these within the 6-month period of the study, and are too young and relatively healthy to be motivated about other health risks. Careful consideration must be given as to how to best support students in interpreting these results, as previous studies indicate that carrier-testing results significantly affect future reproductive decisionmaking, and individuals may have difficulty understanding risk prediction, owing to a lack of understanding of the potential for false-negative results [24-27]. The potential for misinterpretation and the low probability that students will access counseling on a voluntary basis point to the need for new models for clinical consultation and clear institutional policies regarding counseling of student populations in the context of coursework. 
Our results also indicate that students may need new models for technical consultation with instructors or other experts who could answer questions about the application of bioinformatics tools to personal genetic data. Students felt that confidentiality guidelines prohibited instructors from discussing and clarifying individual results. Their desire for more individualized attention reflects a tendency by students to conflate the aim of using personal genotyping in coursework to learn general concepts in human genetics with an opportunity to interpret their own personal genotype data. Recognizing the student inclination to mine their personal genome, this study indicates a potential need for technical consultants who are not responsible for coursework evaluations and who could offer confidential expertise to students who choose to use the knowledge they learned in the course to analyze their own data. Given the wide availability of web-based tools and platforms, it is reasonable to expect that students will probe their raw data for information on variants beyond what is given to them by a particular testing service. Harris et al. [28] have identified the expanding role of genetic counseling in the context of DTC genetic testing and the broad range of skills and knowledge domains necessary to assist consumers with their engagement with genotyping results [22]. Focusing solely on providing traditional genetic counseling fails to consider the spectrum of issues that may provoke questions by student participants.

Our study results reveal that student had a limited recollection and understanding of the consent and biobanking agreements, pointing to the benefits of legal and ethical consultants who can act as resources to students unfamiliar with reviewing these documents. Although the informed consent processes in the personal genome-testing industry are shifting [29], clear identification of when customers are merely consumers and when they become research participants remains murky. It is imperative that institutions provide support to students on how to interpret the conditions of their purchase of personal genome-testing services and how to manage the potential longer-term relationship they may have with these companies even after they complete the course and graduate from their programs. A multi-disciplinary approach to consultation services should be sufficiently flexible to address student needs as the landscape of services and quality of information evolves.

\section{Conclusion}

As academic institutions partner with DTC personal genotyping services to create new curricula, careful consideration of student needs and of the support services made available to them is crucial. The experiences and perspectives of students who have been genotyped in the classroom setting should guide educators as they build models for incorporating personal genotyping into medical and graduate curricula. Student perspectives indicate a need for improvements to achieve a more nuanced treatment of the pedagogical needs of the student population in question. In planning a new curriculum, a multi-disciplinary approach, as taken by Stanford University's task force, would help to anticipate the range of topics, including technical, clinical, social and ethical issues, incumbent in offering or encouraging genotyping in a course context. Building on this model, the inclusion of students and/or community members who have experienced genotyping would provide added perspectives in anticipating the emerging needs of student populations. Ongoing, innovative multi-disciplinary consultation support would allow students the opportunity to take full advantage of the learning experience by confirming appropriate use of the tools and receiving individualized attention when confronted with the practical and analytical challenges of interpreting the human genome. These lessons provide a framework for the development of a model for when personal genetic testing is introduced in the classroom while minimizing associated risks to students and beginning the important work of creating best practices. Further research into the experiences of students who are offered genotyping in diverse educational settings and levels is needed to equip institutional policies.

\section{Abbreviations}

DTC: direct-to-consumer; IVF: in vitro fertilization.

\section{Authors' contributions}

SL conceived of the study and carried out all the interviews. SL and SV coded and analyzed the data and drafted the manuscript. KS and KO reviewed and provided comments and edits to the manuscript. All authors read and approved the final manuscript.

\section{Authors' information}

Simone Vernez was the Research Project Manager of the NHGRI-funded R01 grant project, 'Social Networking and Personal Genomics: Emerging Issues for Health Research' at the Stanford Center for Biomedical Ethics. She now attends the University of California at Irvine School of Medicine.

Keyan Salari, M.D., Ph.D., is a surgical resident at the Massachusetts General Hospital and Harvard Medical School. He was an M.D./Ph.D. student in the Department of Genetics and the School of Medicine at Stanford University. Kelly Ormond, M.S., C.G.C., is an Associate Professor and Program Director of the MS in Human Genetics and Genetic Counseling in the Department of Genetics at Stanford University.

Sandra Soo-Jin Lee, Ph.D. is a Senior Research Scholar and a medical anthropologist at the Stanford Center for Biomedical Ethics and faculty in the Program in Science, Technology and Society at Stanford University. She is principal investigator (PI) of the NHGRI-funded R01 grant project, 'Social Networking and Personal Genomics: Emerging Issues for Health Research.

\section{Competing interests}

The authors declare that they have no competing interests.

\section{Acknowledgements}

Funding for this work was provided by NIH 1R01HG005086-01 (PI: SL) and P50 HG003389 (PI: MC). We thank Emily Borgelt, M.A. for her generous assistance in the preparation and editing of this manuscript. 


\section{Author details}

'Stanford Center for Biomedical Ethics, 1215 Welch Road, Mod A, Stanford, CA 94305 USA. ${ }^{2}$ Department of Genetics, Stanford University, Mail Stop-5120 Stanford, CA 94305 USA. ${ }^{3}$ Department of Pediatrics, Division of Medical Genetics, Stanford University School of Medicine, 300 Pasteur Drive, Boswell Building A097, Stanford, CA 94304 USA.

Received: 23 August 2012 Accepted: 19 March 2013

Published: 19 March 2013

\section{References}

1. McGuire A, Burke W: An unwelcome side effect of direct-to-consumer personal genome testing: raiding the medical commons. JAMA 2008, 300:2669-2671.

2. Bloss CS, Schork NL, Topol EJ: Effect of direct-to-consumer genomewide profiling to assess disease risk. New England Journal of Medicine 2011, 364:524-534.

3. Farghar EA, Tricker K, Newman W, Wlliott R, Roberts SA, Shaffer JL, Bruce I, Payne K: Current use of pharmacogenetic testing: a national survey of thiopurine methyltransferase testing prior to azathioprine prescription. Journal of Clinical Pharmacy and Therapeutics 2007, 32:187-195.

4. Cincinnati Childrens Hospital Genetic Pharmacology Service: [http://www. cincinnatichildrens.org/service/g/genetic-pharmacology/drugs-tested/], (accessed 11/12/12).

5. [http://www.harthosp.org/InstituteOfLiving/Pharmacogenetics/default.aspx], (accessed 11/12/12).

6. Childs B, Wiener C, Valle D: A Science of the individual: implications for a medical school curriculum. Annu Rev Genomics Hum Genet 2005, 6:313-30.

7. [http://www.shodair.org/drug-metabolizing-testing.asp], (accessed 11/12/12).

8. Childs B, Wiener C, Valle D: A science of the individual: implications for a medical school curriculum. Annu Rev Genomics Hum Genet 2005, 6:313-30.

9. Salari K: The dawning era of personalized medicine exposes a gap in medical education. PloS Med 2009, 6:e1000138.

10. Haspel RL, Arnaout R, Briere L, Kantarci S, Marchand K, Tonellato P, Connolly J, Boguski MS, Saffitz JE: A call to action: training pathology residents in genomics and personalized medicine. Am J Clin Pathol 2010, 133:832-834.

11. Haspel RL, Arnaout R, Briere L, Kantarci S, Marchand K, Tonellato P, Connolly J, Boguski MS, Saffitz JE: A curriculum in genomics and personalized medicine for pathology residents. Am J Clin Pathol 2010, 133-136.

12. Vence T: Docs brace for genomics. Genome Web [http://www.genomeweb. com/dxpgx/docs-brace-dtc-genomics].

13. Taylor ATS, Rogers JC: Teaching about genetic testing issues in the Undergraduate Classroom: a case study. J Genet Counsel 2011, 20:231-240.

14. Sharp RR, Goldlust ME, Eng C: Addressing gaps in physician education using personal genomics. Genetics in Medicine 2011, 13:750-751.

15. Walt DR, Kuhlik A, Epstein SK, Demmer LA, Knight M, Chelmow D, Rosenblatt M, Bianchi DW: Lessons learned from the introduction of personalized genotyping into a medical school curriculum. Genetics in Medicine 2011, 13:63-66.

16. Burke W, Evans JP: Teaching with single nucleotide polymorphisms learning the right lessons. Genetics in Medicine 2011, 13:17-18.

17. Callier S: Swabbing students: should universities be allowed to facilitate educational DNA testing? American Journal of Biomedical Ethics 2012, 12:32-40.

18. Dean's Newsletter. [http://deansnewsletter.stanford.edu/archive/09_28_09. $h \mathrm{html}$.

19. Guttmacher AE, Perteous ME, Mclnerney JD: Educating health-care professionals about genetics and genomics. Nature Reviews Genetics 2007, 8:151-157.

20. Knoell DL, Johnston JS, Bao S, Kelley KA: A Genotyping exercise for pharmacy practice. American Journal of Pharmaceutical Education 2009, 73, Article 43.

21. Salari K, Pizzo PA, Prober CG: Commentary: to genotype or not to genotype? Addressing the debate through the development of a genomcis and personalized medicine curriculum. Academic Medicine 2011, 86:925-927.

22. Kaufman DJ, Bollinger JM, Dyoskin RL: Risky business: risk perception and the use of medical services among customers of dtc personal genetic testing. J Genetic Counseling 2012, 21-43.
23. Tobin SL, Cho MK, Lee SSJ, Magnus DC, Allyse M, Ormond KE, Garrison NA: Customers or research participants? Guidance for research practices in commercialization of personal genomics. Genetics in Medicine 2012, 14:883-835.

24. Denayer L, Welkenhuysen M, Evers-Kiebooms G, Cassiman JJ, Van den Berghe $\mathrm{H}$ : Risk perception after CF carrier testing and impact of the test result on reproductive decision making. American Journal of Medical Genetics 1997, 69:422-428.

25. Ormond KE, Mills $P L$, Lester LA, Ross LF: The effect of family history on disclosure patterns of cystic fibrosis carrier status. American Journal of Medical Genetics 2003, 119:70-77.

26. Lewis C, Skirton H, Jones R: Can we make assumptions about the psychosocial impact of living as a carrier based on studies assessing the effects of carrier testing? Journal of Genetic Counseling 2011, 20:80-97.

27. Sivell S, Elwyn G, Gaff C, Clarke AJ, Iredale R, Shaw C, Dundon J, Thornton $\mathrm{H}$, Edwards A: How risk is perceived, constructed and interpreted by clients in clinical genetics and the effects on decision making: systemic review. Journal of Genetic Counseling 2008, 17:30-63.

28. Harris A, Kelly SE, Wyatt $\mathrm{S}$ : Counseling customers: emerging roles for genetic counselors in the direct-to-consumer genetic testing market. J Genetic Counseling 2012.

29. Gene 210 Home Page. [http://stanford.edu/class/gene210/web/html/ welcome.html].

\section{doi:10.1186/gm428}

Cite this article as: Vernez et al.: Personal genome testing in medical education: student experiences with genotyping in the classroom. Genome Medicine 2013 5:24.

\section{Submit your next manuscript to BioMed Central and take full advantage of:}

- Convenient online submission

- Thorough peer review

- No space constraints or color figure charges

- Immediate publication on acceptance

- Inclusion in PubMed, CAS, Scopus and Google Scholar

- Research which is freely available for redistribution

Submit your manuscript at www.biomedcentral.com/submit
C BioMed Central 